\title{
Efecto de diferentes proporciones de luz LED azul:roja en plantas de chile habanero (Capsicum chinense Jacq.)
}

\author{
Effect of different blue:red LED light ratios on habanero pepper (Capsicum chinense Jacq.) plants
}

\author{
Jorge Enrique Mendoza-Paredes'*, Ana María Castillo-González', Edilberto Avitia-García', Luis Alonso Valdéz- \\ Aguilar', María del Rosario García-Mateos' \\ Departamento de Fitotecnia, Universidad Autónoma Chapingo. Km 38.5 Carretera México-Texcoco. 56230, Chapingo, \\ Estado de México, México. \\ 2 Departamento de Horticultura, Universidad Autónoma Agraria Antonio Narro. Calzada Antonio Narro 1923. CP. 25315. \\ Buenavista, Saltillo, Coahuila. México.
}

\section{RESUMEN}

El chile habanero es el cultivo hortícola de mayor importancia económica en la península de Yucatán. Este podría desarrollarse en ambiente controlado con iluminación LED y permitiría tener una producción continua, con un aumento exponencial de rendimiento y estar libre de plagas y enfermedades. Los objetivos fueron evaluar el efecto de cinco proporciones de luz LED azul:roja y un testigo fluorescente sobre crecimiento en planta, concentración de pigmentos fotosintéticos, compuestos fenólicos y nutrimental en hoja; germinación de polen, grosor de hoja, anatomía de ovario y antera de chile habanero 'Mayan Ba'alché' bajo condiciones controladas de crecimiento. Los resultados mostraron que los tratamientos no indujeron un efecto en crecimiento de planta. La luz roja favoreció el rendimiento de frutos y frecuencia estomática. La luz roja monocromática favoreció la concentración de pigmentos fotosintéticos, $\mathrm{Mg}$ y $\mathrm{Mn}$ e indujo menor concentración de P y Cu. La luz azul monocromática favoreció la concentración de compuestos fenólicos. El testigo indujo mayor concentración de $\mathrm{K}$ entre algunos tratamientos LED e incrementó la germinación de polen y el grosor de hoja al igual que la luz azul monocromática. La anatomía de los óvulos en ovarios y el polen en anteras no presentaron ningún efecto por los tratamientos.

Palabras clave: Capsicum chinense Jacq., chile habanero, luz LED, ambiente controlado.

\section{ABSTRACT}

Habanero pepper is the most economically important horticultural crop in the Yucatan peninsula. This crop under a controlled environment with LED lighting, would allow continuous production, with an exponential increase in yield, and be free of pests and diseases. The objectives were to evaluate the effects of five blue:red LED light ratios and a fluorescent control on plant growth, photosynthetic pigments, phenolic compounds and nutriments concentration in leaf; pollen germination, leaf thickness, ovary anatomy and anther of habanero pepper 'Mayan Ba'alché' grown under controlled conditions. The results showed that the treatments did not induce an effect on plant growth. Red light favored fruit yield and stomatal frequency. Monochromatic red light favored the concentration of photosynthetic pigments, $\mathrm{Mg}$ and $\mathrm{Mn}$ and induced a lower $\mathrm{P}$ and $\mathrm{Cu}$ concentration. Monochromatic blue light favored the concentration of phenolic compounds. The control induced a higher $\mathrm{K}$ concentration among some LED treatments and increased pollen germination and leaf thickness as well as monochromatic blue light. The anatomy of ovules in ovaries and pollen in anthers has no effect of the treatments.

Keywords: Capsicum chinense Jacq., habanero pepper, LED light, controlled environment.

\section{INTRODUCCIÓN}

El chile habanero (Capsicum chinense Jacq.) es una planta anual de tipo arbustivo (Lim, 2013) nativa de las tierras bajas de la cuenca amazónica, donde se dispersó en la época prehispánica a la cuenca del Orinoco y otras regiones de Perú, las Antillas y finalmente arribó a la Península de Yucatán (Trujillo, 2018). Actualmente este es el cultivo hortícola de mayor importancia económica para la península de Yucatán en México (Ruiz-Lau et al., 2011); desde 2010 posee la denominación de origen en esta región (CICY, 2016). El fruto posee diversas propiedades nutraceúticas y medicinales (Lim, 2013), es usado también en la industria alimentaria, farmacéutica y militar (Ruiz-Lau et al., 2011).

Debido a esto, ha surgido la necesidad de realizar investigación de su cultivo en ambiente controlado con iluminación LED para analizar el comportamiento como una alternativa de producción, ya que con este sistema se elimina la aparición de plagas y enfermedades, por lo tanto, la obtención de un producto orgánico libre de plaguicidas e inocuo a la salud humana. Otra ventaja sería su producción continua, sin estacionalidad de cultivo, por el control de las variables ambientales y de la fuente de luz, lo cual en su conjunto trae un incremento exponencial de la producción (Kozai y Niu, 2016).

Actualmente no existe investigación de esta especie bajo estas condiciones de crecimiento; sin embargo, se han realizado investigaciones por parte de Gangadhar et al. (2012) en chile picante cv. Cheonyang y Naznin et al. (2019) en pimiento dulce enano cv. Redstart, en ambas investigaciones existieron diferencias entre los tratamientos de luz LED azul y roja que evaluaron, respecto a las variables del crecimiento como altura de planta, área foliar, peso fresco de la parte aérea, número de frutos por planta y en los pigmentos fotosintéticos en hoja como clorofila $a, b$, total $a / b$ y carotenoides.

*Autor para correspondencia: Jorge Enrique Mendoza Paredes Correo electrónico:jmendozaparedes@hotmail.com

Recibido: 9 de mayo de 2020 Aceptado: 24 de agosto de 2020 
Con base en la información anterior, el objetivo del presente estudio fue evaluar el efecto de diferentes proporciones de luz LED roja y azul sobre crecimiento, concentración de pigmentos fotosintéticos, fenoles totales y nutrimental en hoja; así como determinar si los tratamientos influyen en la germinación de polen, grosor de hoja, frecuencia estomática, anatomía de ovario y antera de chile habanero.

\section{MATERIALES Y MÉTODOS Cámara y gabinetes de crecimiento}

Se realizó una adaptación de una cámara de crecimiento a partir de un cuarto frío (TORREY ${ }^{\circledast}, \mathrm{MEX}$ ), con medidas interiores de $2.76 \mathrm{~m} \times 2.76 \mathrm{~m} \times 1.90 \mathrm{~m}$ (ancho, largo y altura), las modificaciones internas de la cámara de crecimiento se basaron en el diseño de Katagiri et al. (2015), se reguló la temperatura ambiental y humedad relativa con un equipo de aire acondicionado portátil (LG ${ }^{\oplus}$ modelo LP1017WSR 10200 BTU, KOR) y un deshumidificador (Whirlpool ${ }^{\circledR}$ modelo WAD200 5Q $20 \mathrm{~L} / 24 \mathrm{~h}, \mathrm{USA}$ ). En el interior de esta cámara, se diseñaron seis gabinetes de crecimiento a partir de un anaquel metálico de $0.3 \mathrm{~m} \times 0.85 \mathrm{~m} \times 1.85 \mathrm{~m}$ (ancho, largo y altura) a los cuales se les añadieron paredes de placas de unicel de $2 \mathrm{~cm}$ de grosor forradas con papel aluminio para tener máxima reflexión de la luz en el interior y para aislar los tratamientos uno de otro.

\section{Tratamientos con diferentes proporciones de luz LED azul y roja}

Las luminarias que se utilizaron fueron hechas a mano, compuestas por perfiles de aluminio de $5 \mathrm{~cm} \times 85$ $\mathrm{cm}$ (ancho $\mathrm{x}$ largo) en donde se anclaron focos LED azul y rojos (Longniu ${ }^{\circledR}$ modelo GR-DB015 de 4 watts, $\mathrm{CHN}$ ) y las lámparas fluorescentes (Osram ${ }^{\circledR}$ modelo Octron/F017 de 17 watts, DEU). Las luminarias fueron colocadas debajo de la parte superior de cada uno de los gabinetes de crecimiento. Los tratamientos consistieron en un testigo fluorescente (4 lámparas) y en los tratamientos A0 \%:R100 \% (A0) con 24 focos LED rojos, A34.5 \%:R65.5 \% (A34.5) con 18 focos LED rojos y 6 azules, A57.1\%:R42.9\% (A57.1) con 12 focos LED rojos y 12 azules, A76 \%:R24 \% (A76) con 6 focos LED rojos y 18 azules, A100 \%:R0 \% (A100) con 24 focos LED azules. Se utilizaron dos perfiles por tratamiento ( 12 focos LED por perfil) y el testigo con cuatro perfiles ( 1 lámpara fluorescente por perfil). La densidad de flujo de fotones fotosintéticos (DFFF) $\left(\mu \mathrm{mol} \mathrm{m}^{2} \mathrm{~s}^{-1}\right)$ y las propiedades físicas del espectro electromagnético de los tratamientos se midió con un espectroradiómetro (Apogee instruments ${ }^{\circledR}$ modelo SS-110, EUA) donde se tomaron 330 mediciones sobre el área de cultivo $(66 \mathrm{~cm} \mathrm{x}$ $20 \mathrm{~cm}=1320 \mathrm{~cm}^{2}$ ), que fue la superficie en relación a la parte inferior del gabinete, donde las plantas tuvieron su desarrollo durante el transcurso del experimento, y así verificar que los tratamientos tuvieran la misma densidad de flujo de fotones fotosintéticos (DFFF). Se realizó un análisis estadístico de las variables físicas de los tratamientos (Tabla 1) para asegurar que las intensidades de luz entre tratamientos LED fueran iguales y las proporciones de luz azul y roja fueran diferentes; además de verificar el pico de longitud de onda (nm) e intervalo de la luz LED presente en los tratamientos. Se estableció un fotoperiodo para todos los tratamientos de luz de $15 \mathrm{~h}$ por día durante 145 días después del trasplante.

\section{Material vegetal}

Se utilizaron semillas de chile habanero 'Mayan Ba'alché' del CICY (Centro de Investigación Científica de Yucatán), las cuales previamente se sembraron en un semi-

Tabla 1. Propiedades físicas de los tratamientos con diferentes proporciones de luz LED azul y roja en chile habanero cultivado bajo condiciones controladas.

Table 1. Physical properties of treatments with different blue and red LED light ratios on habanero pepper grown under controlled conditions.

\begin{tabular}{|c|c|c|c|c|c|c|}
\hline & \multicolumn{6}{|c|}{ Tratamientos $^{\mathrm{x}}$} \\
\hline & $\mathbf{F L}$ & AO & A34.5 & A57.1 & A76.0 & A100 \\
\hline $\operatorname{PrA}$ & Testigo & AO \% & A34.5\% & A57.1\% & A76 \% & A100 \% \\
\hline PrR & Testigo & R100 \% & R65.5 \% & R42.9\% & $\mathrm{R} 25.8 \%$ & Ro \% \\
\hline Dtot & $119.1 \pm 0.6 b$ & $241.5 \pm 3.1 a$ & $241.4 \pm 3.3 a$ & $244.4 \pm 2.4 a$ & $241.4 \pm 1.8 a$ & $243.8 \pm 2.5 a$ \\
\hline LID & $6.4 \pm 0.0 b$ & $13.0 \pm 0.2 \mathrm{a}$ & $13.0 \pm 0.2 a$ & $13.2 \pm 0.1 \mathrm{a}$ & $13.0 \pm 0.1 a$ & $13.2 \pm 0.1 \mathrm{a}$ \\
\hline$\lambda \mathrm{A}$ & - & - & $457 \pm 0 b$ & $457 \pm 0 a$ & $457 \pm 0 b$ & $457 \pm 0 b$ \\
\hline$\% D A$ & - & - & $34.5 \pm 0.6 d$ & $57.1 \pm 0.3 c$ & $76.0 \pm 0.3 b$ & $100.0 \pm 0.0 a$ \\
\hline$\lambda R$ & - & $636 \pm 0 b$ & $635 \pm 0 c$ & $637 \pm 0 a$ & $636 \pm 0 b$ & - \\
\hline$\% D R$ & - & $100.0 \pm 0.0 a$ & $65.5 \pm 0.6 b$ & $42.9 \pm 0.3 c$ & $24.0 \pm 0.3 d$ & - \\
\hline
\end{tabular}

'Medias con letras iguales en las filas son estadísticamente iguales (Tukey, $\mathrm{P} \leq 0.05$ ). FL= fluorescente con un intervalo de 416nm - 715nm (testigo); $\mathrm{A}=$ Luz LED azul; R= Luz LED roja; PrA= Proporción luz azul; PrR= Proporción luz roja; Dtot= Densidad de flujo de fotones fotosintéticos total de tratamiento $\left(\mu \mathrm{mol} \mathrm{m} \mathrm{m}^{-2} \mathrm{~s}^{-1}\right) ; \mathrm{LID}=\mathrm{Luz}$ integral diaria $\left(\mathrm{mol} \mathrm{m}^{-2} \mathrm{~d}^{-1}\right) ; \lambda A=$ Pico de longitud de onda $(\mathrm{nm})$ color azul; \%DA= Porcentaje de densidad de flujo de fotones fotosintéticos de luz azul (\%); $\lambda R=$ Pico de longitud de onda $(\mathrm{nm})$ color rojo; \%DR= Porcentaje de densidad de flujo de fotones fotosintéticos de luz roja (\%). 
llero de 200 cavidades, se colocó una semilla por cavidad a una profundidad de $1 \mathrm{~cm}$ en una mezcla de turba rubia (Pro $\mathrm{mix}^{\oplus}, \mathrm{CAN}$ ) y perlita (Agrolita ${ }^{\oplus}, \mathrm{MXN}$ ) en proporción $3: 1 \mathrm{v} / \mathrm{v}$, los semilleros se cortaron para tener 80 cavidades (4 filas $\times 20$ columnas) y tener una charola por tratamiento. Una vez sembradas, se colocaron en un laboratorio donde tuvieron luz solar incidente a través de una ventana durante la duración del día y fueron regadas con agua potable $(\mathrm{pH}$ 7.1 y CE $0.42 \mathrm{dS} \mathrm{m}^{-1}$ ). La temperatura ambiental (día $25.8 \pm 0.2$ ${ }^{\circ} \mathrm{C} /$ noche $22.6 \pm 0.1{ }^{\circ} \mathrm{C}$ ) y la humedad relativa (día $51.5 \pm 0.6$ $\% /$ noche $58.6 \pm 0.6 \%$ ); se registraron con un datalogger (Extech instruments ${ }^{\oplus}$ modelo RH10, USA). A los 17 días en estas condiciones, al inicio del desarrollo, las plántulas contaban con dos cotiledones y dos hojas; en este momento las charolas con las plántulas se trasladaron a la cámara de crecimiento, para iniciar los tratamientos con luz. En estas nuevas condiciones las plántulas crecieron por 35 días a una temperatura ambiental $\left({ }^{\circ} \mathrm{C}\right.$ ) promedio (día $28.2 \pm 0.1 /$ noche $25.3 \pm 0.1$ ) y humedad relativa (\%) promedio (día $56.2 \pm 0.5 /$ noche $77.1 \pm 0.5$ ). El riego se hizo diariamente a cada una de las charolas con $300 \mathrm{~mL}$ de la solución de Hoagland modificada a 1/5 de su concentración (Bojórquez-Quintal et al., 2014) con $\mathrm{pH}$ de 6.8 y CE de $1.35 \mathrm{dS} \mathrm{cm}^{-1}$, donde se abastecieron los macronutrientes de la forma siguiente: $1.2 \mathrm{mM} \mathrm{KNO}_{3^{\prime}} 0.8 \mathrm{mM}$ $\mathrm{Ca}\left(\mathrm{NO}_{3}\right)_{2^{\prime}} 0.2 \mathrm{mM} \mathrm{KH}_{2} \mathrm{PO}_{4^{\prime}} 0.2 \mathrm{mM} \mathrm{MgSO}_{4}$ y los micronutrientes se abastecieron con la mezcla Tradecorp ${ }^{\circledR} \mathrm{AZ}\left(0.065 \mathrm{~g} \mathrm{~L}^{-1}\right)$.

\section{Trasplante}

Una vez que transcurrieron 35 días, se obtuvieron plántulas adecuadas para trasplante, se eligieron tres por tratamiento y se trasplantó una por maceta, las cuales conservaron los mismos tratamientos de luz (Tabla 1) una vez trasplantadas, se mantuvo así por 145 días después del trasplante (ddt). Se ocuparon tres macetas de $5 \mathrm{~L}$ por tratamiento con una mezcla de turba:perlita (3:1), turba rubia (Pro mix ${ }^{\circledast}$, CAN) y perlita (Agrolita $\left.{ }^{\oplus}, M X N\right)$ y se regaron diariamente con $1 \mathrm{~L}$ de la solución nutritiva de Hoagland (Hoagland y Arnon, 1950) a $100 \%$ de concentración de macronutrientes con un pH de 5.8 y CE de $1.33 \mathrm{dS} \mathrm{cm}^{-1}$, los micronutrientes se abastecieron con la mezcla Tradecorp ${ }^{\oplus} \mathrm{AZ}\left(0.065 \mathrm{~g} \mathrm{~L}^{-1}\right)$. La temperatura ambiental promedio fue (día $26.7 \pm 0.1^{\circ} \mathrm{C} /$ noche $23.9 \pm 0.1^{\circ} \mathrm{C}$ ) y la humedad relativa promedio (día $66.1 \pm 0.2 \%$ / noche $82.3 \pm 0.2 \%$ ), éstas se registraron cada minuto con el promedio de tres datalogger (Extech instruments ${ }^{\circledR}$ modelo $\mathrm{RH} 10, \mathrm{USA})$, los cuales se colocaron uno por gabinete a nivel de copa de la planta en los tratamientos A76 \%:R24 \%, A34.5 \%:R65.5 \% y el testigo. La temperatura promedio de la solución nutritiva se midió con un termómetro infrarrojo (Extech instruments ${ }^{\circledR}$ modelo 42530, USA) y tuvo un valor de $26.2 \pm 1.3^{\circ} \mathrm{C}$. La concentración promedio de $\mathrm{CO}_{2}$ se midió con un medidor portátil de $\mathrm{CO}_{2}$ ambiental (Extech instruments ${ }^{\circledast}$ modelo CO250, USA) y tuvo una media de $404 \pm 52$ ppm. Durante el crecimiento de la planta se realizaron podas de chupones y ramas para tener una planta con dos tallos, además se realizaron podas de hojas en la parte inferior de la planta con el fin de evitar un sombreo excesivo entre éstas.
También se realizaron polinizaciones manuales con un pincel para aumentar el porcentaje de amarre de frutos.

\section{Variables de crecimiento de la planta}

A los 145 (ddt), las plantas se cosecharon y se determinó la altura de planta $(\mathrm{cm})$, el peso fresco de tallo y de hoja (g planta ${ }^{-1}$ ), con una balanza de precisión (Ohaus ${ }^{\circledR}$ Scout Pro, USA), el peso fresco de la parte aérea ( $\mathrm{g} \mathrm{planta}^{-1}$ ) se estimó con la suma del peso fresco del tallo y de las hojas. El diámetro de tallo $(\mathrm{mm})$ se midió con un vernier digital (Truper ${ }^{\circledast}, \mathrm{MEX}$ ); el área foliar $\left(\mathrm{cm}^{2}\right.$ planta-1 $)$ se determinó con un integrador de área foliar LI-3100 (LICOR ${ }^{\circledR}$, USA) y se calculó el índice de área

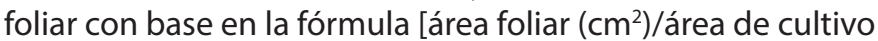
$\left.\left(\mathrm{cm}^{2}\right)\right]$. La parte aérea de la planta se secó en una estufa de aire forzado (BINDER ${ }^{\circledR}$ modelo FED 115, DEU) por 48 horas a $65^{\circ} \mathrm{C}$ y se registró el peso seco de tallo y hoja (g planta-1), ambos por separado, con una balanza de precisión (Ohaus ${ }^{\circledR}$ Golden series, USA), el peso seco de la parte aérea ( g planta $^{-1}$ ) se determinó mediante la suma del peso seco del tallo y de las hojas. Los frutos se cosecharon a los 109 y $145 \mathrm{ddt}$ y se contabilizó el total por tratamiento. La frecuencia estomática (número de estomas por $\mathrm{mm}^{2}$ ) se determinó mediante la aplicación de barniz transparente sobre el envés de la hoja; posteriormente, se despegó la calca que se formó en la hoja y se colocó en un portaobjetos para la contabilización de los estomas con un microscopio (Carl Zeiss ${ }^{\circledast}$ Axiostar, DEU), con aumento de $40 \mathrm{x}$. En cada una de las variables medidas se utilizaron tres repeticiones por tratamiento, excepto para la frecuencia estomática donde se tuvieron 10 repeticiones.

\section{Concentración de pigmentos fotosintéticos en hoja}

Las concentraciones de clorofila a, b y total ( $\mathrm{mg} \mathrm{g}^{-1}$ de peso fresco), proporción de clorofila a/b y concentración de carotenoides ( $\mathrm{mg} \mathrm{g}^{-1}$ de peso fresco) se determinaron mediante la metodología descrita por la AOAC (1980). Estas variables se determinaron en hojas fuente (de reciente maduración), completamente desarrolladas, recién cortadas y utilizando tres hojas por planta por tratamiento.

\section{Compuestos fenólicos totales en hoja}

Se tomaron $2 \mathrm{~g}$ de hojas fuente completamente desarrolladas recién cortadas, las cuales se cortaron finamente y se les adicionó $10 \mathrm{ml}$ de $\mathrm{MeOH}$ al $80 \%(\mathrm{v} / \mathrm{v})$, posteriormente se homogeneizó mediante sonicación por 15 minutos a temperatura ambiente y se dejó reposar durante $24 \mathrm{~h}$ en refrigeración y oscuridad. Pasado este tiempo, se tomó $0.1 \mathrm{~mL}$ del extracto metanólico previamente preparado, se agregó $0.1 \mathrm{~mL}$ del reactivo Folin-Ciocalteu $0.2 \mathrm{~N}, 4.5 \mathrm{ml}$ agua destilada y $0.3 \mathrm{ml} \mathrm{de} \mathrm{Na}_{2} \mathrm{CO}_{3} 2 \%(\mathrm{p} / \mathrm{v})$, esta mezcla se incubó a temperatura ambiente y en oscuridad por $2 \mathrm{~h}$. Posteriormente se tomaron lecturas de absorbancia del sobrenadante en un espectrofotómetro Genesys 10s (Thermo Fisher Scientific, USA) a una longitud de onda de $765 \mathrm{~nm}$. La concentración de compuestos fenólicos se calculó a partir de una curva estándar preparada con ácido gálico y se expresó en $\mathrm{mg}$ equivalentes de ácido gálico por $100 \mathrm{~g}$ de peso fresco 
(mg EAG $100 \mathrm{~g}^{-1} \mathrm{PF}$ ) de acuerdo con el método de Singleton y Rossi (1965) con algunas modificaciones. Se utilizaron tres repeticiones de cada tratamiento.

\section{Concentración nutrimental en hojas}

Se determinaron $\mathrm{P}, \mathrm{K}, \mathrm{Ca}, \mathrm{Mg}, \mathrm{Fe}, \mathrm{Mn}, \mathrm{Zn}, \mathrm{B}$ y $\mathrm{Cu}$ en un espectrofotómetro de Emisión Atómica de Plasma por Inducción Acoplada (ICP-AES) (VARIAN modelo Liberty II, USA); la concentración de $\mathrm{N}$ se determinó con el método micro-Kjeldahl (Alcántar y Sandoval, 1999). Se utilizaron para las determinaciones $0.1 \mathrm{~g}$ de hojas fuente completamente desarrolladas previamente secas en una estufa de aire forzado (BINDER ${ }^{\circledast}$ modelo FED 115, DEU) por 48 horas a 65 ${ }^{\circ} \mathrm{C}$. Para la determinación de los elementos se utilizaron tres repeticiones por tratamiento.

\section{Variables anatómicas y germinación de polen}

A los 106 ddt, se realizaron cortes anatómicos longitudinales de flores en antesis y cortes transversales de hojas completamente desarrolladas. Por tratamiento se utilizaron cinco flores y cinco hojas. En el caso de las flores, se eliminaron los pétalos y posteriormente se seccionó el ovario a la mitad de manera longitudinal con un bisturí. Para el caso de las hojas se tomó una sección de $5 \times 5 \mathrm{~mm}$ por hoja, con ayuda de un bisturí, de la parte media de la hoja que se ubicó entre la vena central y el borde. Tanto para el caso de los ovarios como de hojas se fijaron en FAA (50 \% etanol al 96 $\%+5 \%$ ácido acético glacial + $10 \%$ formaldehído al $37 \%+$ $35 \%$ de agua). Posteriormente se realizó un lavado en agua y etanol al $70 \%$ y a continuación se conservaron en GAA ( 25 $\%$ glicerol $+50 \%$ 2-propanol $+0.002 \%$ eosina $+25 \%$ agua) hasta el momento de incluirlos en parafina para realizar los cortes anatómicos que tuvieron un grosor de $10 \mu \mathrm{m}$, para lo cual se utilizó un micrótomo rotatorio. La observación se realizó en un microscopio (Carl Zeiss ${ }^{\circledR}$ Axiostar, DEU) con aumentos de 10X y 40X para ovario más anteras y de 40X para hoja. El grosor de la hoja se midió usando micrómetros objeto y de ocular. Se evaluó la anatomía de los óvulos en el ovario en base a la presencia o ausencia de óvulos, pared del ovario, núcleo del óvulo y tegumentos. Por otra parte, en la anatomía de polen en anteras, se determinó la viabilidad del polen en base al grado de coloración por parte de la tinción sobre el citoplasma de éste, en donde una tinción completa y de color rojo intenso es indicativo de un polen viable, por otra parte, un citoplasma no coloreado, o de una tonalidad baja es indicativo de un polen no viable.

Para la viabilidad de polen se usó la técnica modificada de Mercado et al. (1994), se preparó un medio sólido con $6 \mathrm{~g}$ $\mathrm{L}^{-1}$ de agar-agar, $10 \%$ de sacarosa, $0.1 \mathrm{mM}$ de ácido bórico y 1 $\mathrm{mM}$ de cloruro de calcio para dos cajas Petri. Se recolectaron cinco flores frescas en estado inicial de apertura a antesis a los $135 \mathrm{ddt}$, excepto el tratamiento A100 \%:R0 \% que ya no contaba con flores, el polen fresco de las anteras se depositó sobre la superficie del medio de agar. Las cajas Petri se colocaron dentro de sus respectivos tratamientos de luz por dos horas, se observaron en un microscopio (Carl Zeiss ${ }^{\circledR}$ Axiostar,
DEU) con un aumento de 40X y se calculó el porcentaje de germinación de polen en cuatro campos por caja Petri.

\section{Diseño experimental y análisis estadístico}

El experimento se estableció en un diseño completamente al azar, una repetición estuvo constituida por una plántula. Los datos se sometieron a un análisis de varianza (ANOVA), a la prueba de comparación de medias de Tukey ( $P$ $<0.05$ ) utilizando el programa SAS V9.0 (SAS Institute, 2002).

\section{RESULTADOS Y DISCUSIÓN \\ Variables de crecimiento de la planta}

Los resultados de las variables de crecimiento se muestran en la Tabla 2. Se observó que las plantas que recibieron tratamientos con luz LED la altura de planta no fue significativamente diferente entre estos, sin embargo, el tratamiento con $100 \%$ de luz azul fue mayor al testigo. Las plantas del tratamiento A100, en donde no se recibió ninguna cantidad de luz roja, presentaron la menor frecuencia estomática respecto a las plantas que si recibieron cierto porcentaje de luz roja. Bajo la presencia de luz LED roja en los tratamientos evaluados, se obtuvieron frutos, excepto en las plantas que recibieron $34.5 \%$ de luz azul, mientras que en las plantas del tratamiento monocromático rojo se obtuvo la mayor cantidad de frutos. Las variables que resultaron estadísticamente no significativos pudieron deberse a las podas de hojas a las cuales fueron sometidas las plantas durante el ciclo de crecimiento.

Verma et al. (2018) encontraron la mayor altura de planta en cultivo de digitales con un proporción de luz LED azul y roja $\mathrm{A} 100 \%: \mathrm{R} 0 \%$ respecto a un tratamiento testigo fluorescente. Por otra parte, Naznin et al. (2019) no encontraron diferencias estadísticas en altura de planta de espinaca y albahaca entre cuatro diferentes tratamientos de luz roja y azul (A17 \%:R83 \%, A9 \%:R91 \%, A5 \%:R95 \%, A0 \%:R100 \%). Las plantas del testigo, a pesar de que recibieron la mitad de la intensidad luminosa en comparación con los tratamientos LED (Tabla 1), no tuvieron diferencia en altura de planta con los tratamientos LED que tuvieron presencia de luz roja, sin embargo, con las plantas que recibieron $100 \%$ de luz azul si fue menor.

La luz roja a través de la acción de los fitocromos, tiende a elongar la altura de las plantas, mientras que la luz azul se le ha asociado con la formación más compacta y de menor altura en plantas (Berkovich et al., 2017; Pocock, 2015). Sin embargo, en habanero, al no existir diferencias significativas entre tratamientos LED, la luz roja o azul no tuvieron un efecto evidente sobre la altura de planta, salvo en el caso de las plantas del testigo en comparación con las que recibieron $100 \%$ de luz azul, la diferencia de altura pudo deberse principalmente a la menor intensidad luminosa que presentó.

Wollanger y Runkle (2015) encontraron un comportamiento similar en el área foliar en plántula de tomate. El índice de área foliar debe encontrarse entre 3.0 a 4.0 para que se aproveche de un 90 a $100 \%$ de luz incidente, si es mayor el valor como en los resultados obtenidos, supone una pérdida 
Tabla 2. Variables de crecimiento evaluadas en chile habanero cultivado bajo condiciones controladas con diferentes proporciones de luz LED azul y roja.

Table 2. Growth variables evaluated in habanero pepper grown under controlled conditions with different ratios of blue and red LED light.

\begin{tabular}{|c|c|c|c|c|c|c|}
\hline \multirow[b]{2}{*}{ Var } & \multicolumn{6}{|c|}{ Tratamientos $^{x}$} \\
\hline & FL & AO & A34.5 & A57.1 & A76.0 & A100 \\
\hline AP & $85.50 \pm 10.28 b$ & $102.90 \pm 35.52 \mathrm{~b}$ & $94.63 \pm 11.66 b$ & $97.37 \pm 17.41 b$ & $109.70 \pm 17.97 b$ & $176.77 \pm 4.57 a$ \\
\hline GT & $8.03 \pm 0.28^{\mathrm{ns}}$ & $10.91 \pm 1.71^{\mathrm{ns}}$ & $11.30 \pm 1.40^{\mathrm{ns}}$ & $11.95 \pm 0.73^{\mathrm{ns}}$ & $9.05 \pm 0.85^{\mathrm{ns}}$ & $9.80 \pm 0.33^{\mathrm{ns}}$ \\
\hline $\mathrm{AF}$ & $2008.0 \pm 714.2^{\mathrm{ns}}$ & $1991.0 \pm 1592.3^{\text {ns }}$ & $2703.0 \pm 1037.3^{\text {ns }}$ & $2661.0 \pm 828.0^{\text {ns }}$ & $2222.0 \pm 1220.5^{n}$ & $1989.0 \pm 565.1^{\mathrm{n}}$ \\
\hline IAF & $4.56 \pm 1.62^{\mathrm{ns}}$ & $4.56 \pm 3.62^{\mathrm{ns}}$ & $6.16 \pm 2.36^{\mathrm{ns}}$ & $6.06 \pm 1.88^{\text {ns }}$ & $5.03 \pm 2.77^{\mathrm{ns}}$ & $4.53 \pm 1.28^{\text {ns }}$ \\
\hline PFT & $47.00 \pm 12.45^{\mathrm{ns}}$ & $74.73 \pm 48.50^{\mathrm{ns}}$ & $81.27 \pm 26.48^{\text {ns }}$ & $61.03 \pm 9.80^{\mathrm{ns}}$ & $80.00 \pm 48.66^{\mathrm{ns}}$ & $182.63 \pm 45.76^{n}$ \\
\hline PFH & $34.27 \pm 4.64^{\mathrm{ns}}$ & $36.50 \pm 11.11^{\mathrm{ns}}$ & $49.90 \pm 7.47^{\mathrm{ns}}$ & $56.23 \pm 7.41^{\mathrm{ns}}$ & $44.83 \pm 10.10^{\text {ns }}$ & $44.97 \pm 6.74^{\mathrm{ns}}$ \\
\hline PFPA & $81.27 \pm 23.82^{\mathrm{ns}}$ & $111.23 \pm 76.79^{\text {ns }}$ & $131.17 \pm 45.77^{\mathrm{ns}}$ & $117.27 \pm 14.82^{\mathrm{ns}}$ & $124.83 \pm 74.08^{\mathrm{ns}}$ & $227.60 \pm 62.48^{\text {n }}$ \\
\hline PST & $6.49 \pm 2.36^{\mathrm{ns}}$ & $13.68 \pm 9.50^{\mathrm{ns}}$ & $12.52 \pm 5.96^{\mathrm{ns}}$ & $12.87 \pm 3.95^{\mathrm{ns}}$ & $12.41 \pm 8.82^{\text {ns }}$ & $22.43 \pm 6.67^{\mathrm{ns}}$ \\
\hline PSH & $3.31 \pm 1.55^{\mathrm{ns}}$ & $4.17 \pm 3.66^{\mathrm{ns}}$ & $5.04 \pm 2.58^{\text {ns }}$ & $5.78 \pm 2.30^{\text {ns }}$ & $5.62 \pm 3.76^{\mathrm{ns}}$ & $5.65 \pm 2.51^{\mathrm{ns}}$ \\
\hline PSPA & $9.80 \pm 1.51^{\mathrm{ns}}$ & $17.85 \pm 5.10^{\text {ns }}$ & $17.55 \pm 3.31^{\mathrm{ns}}$ & $18.65 \pm 2.38^{\mathrm{ns}}$ & $18.03 \pm 4.86^{\mathrm{ns}}$ & $28.07 \pm 3.52^{\mathrm{ns}}$ \\
\hline $\mathrm{FE}$ & $127.00 \pm 5.12 b$ & $144.70 \pm 7.32 \mathrm{ab}$ & $128.30 \pm 6.30 \mathrm{ab}$ & $154.00 \pm 11.57 a b$ & $159.30 \pm 7.14 a$ & $88.30 \pm 6.76 c$ \\
\hline NF & 0 & 24 & 0 & 5 & 4 & 0 \\
\hline
\end{tabular}

×Medias con igual letra en las filas son estadísticamente iguales (Tukey, $\mathrm{P} \leq 0.05)$. $\mathrm{ns}=$ No significativo; $\mathrm{Var}=\mathrm{Variables;} \mathrm{FL}=$ fluorescente (testigo); $A P=$ Altura de planta $(\mathrm{cm}) ; \mathrm{GT}=\mathrm{Grosor}$ de tallo $(\mathrm{mm}) ; \mathrm{AF}=$ Área foliar $\left(\mathrm{cm}^{2}\right.$ planta $\left.{ }^{-1}\right) ; \mathrm{IF}=$ Índice de área foliar; PFT= Peso fresco de tallo $\left(g_{\text { planta }}{ }^{-1}\right) ; \mathrm{PFH}=$ Peso fresco de hoja $\left(\mathrm{g}\right.$ planta-1 $\left.{ }^{-1}\right) ; \mathrm{PFPA}=$ Peso fresco de la parte aérea $\left(\mathrm{g}\right.$ planta $\left.{ }^{-1}\right) ; \mathrm{PST}=$ Peso seco de tallo $\left(\mathrm{g} \mathrm{planta}^{-1}\right) ; \mathrm{PSH}=$ Peso seco de hoja $\left(\mathrm{g}\right.$ planta $\left.{ }^{-1}\right) ; \mathrm{PSPA}=$ Peso seco de la parte aérea $\left(\mathrm{g}\right.$ planta $\left.{ }^{-1}\right) ; \mathrm{FE}=$ Frecuencia estomática $\left(\mathrm{mm}^{2}\right) ; \mathrm{NF}=$ Número de frutos por tratamiento.

de fotosintatos asimilados debido a la existencia de una mayor respiración por causa del sombreo en hojas inferiores de la planta Yamori (2016).

Hogewoning et al. (2010) encontraron en pepino resultados similares en la frecuencia estomática, con tratamientos de $0 \%, 15 \%, 30 \%, 50 \%$ de luz LED azul respecto a la luz LED roja donde no tuvieron diferencias estadísticas entre éstos. De manera contraria Wang et al. (2016) encontraron que el tratamiento monocromático rojo obtuvo la menor frecuencia estomática en lechuga en comparación con diferentes proporciones de luz LED azul y roja. El desarrollo de los estomas se ha asociado a los fitocromos, los cuales son activados por luz roja (Demotes-Mainard et al., 2016; Pocock, 2015), con lo cual, se confirma que en plantas de habanero que recibieron diferentes proporciones de luz LED roja junto con el testigo, que posee un espectro completo de luz (416$715 \mathrm{~nm}$ ), existió una mayor frecuencia estomática que las plantas que recibieron $100 \%$ de luz azul.

Gangadhar et al. (2012) encontraron en chile picante $\mathrm{cv}$. Cheonyang el mayor número de frutos por planta con una proporción de luz azul y roja de A50 \%:R50 \% (3.8), seguido del testigo fluorescente (3.3) y el tratamiento A0 \%:R100 \% (3.0), el menor número se obtuvo con el tratamiento A100 \%:R0 \% (2.1). En el caso de Naznin et al. (2019) registraron en pimiento dulce enano cv. Redstart, el mayor número de frutos por planta con una proporción de luz azul y roja de A5 \%:R95 \% (5), A9 \%:R91 \% (5) y los menores con los tratamientos $A 17 \%: R 83 \%$ (1) y A0 \%:R100 \% (1).
El rendimiento de frutos del testigo pudo haber sido afectado debido a que tuvo la mitad de la luz integral diaria (LID) (6.4 mol m $\mathrm{m}^{-2} \mathrm{~d}^{-1}$ de fotones) en comparación con los tratamientos con luz LED (13.0 a $13.2 \mathrm{~mol} \mathrm{~m}^{-2} \mathrm{~d}^{-1}$ de fotones) (Tabla1). Además, fue $82 \%$ menos de lo necesario para el testigo y $63 \%$ para los tratamientos LED respecto a la LID que requiere una planta de tomate en etapa de producción (35 mol m-2 $\mathrm{d}^{-1}$ de fotones) (Kubota, 2016). Lo anterior pudo generar una baja tasa en la fotosíntesis y una baja concentración de fotosintatos en la planta para llevar a cabo un adecuado amarre de frutos. A pesar de la falta de LID, en las plantas que recibieron solo luz roja existió una mayor cantidad de frutos. Esto pudo deberse a un posible efecto de la luz roja sobre los fitocromos en el amarre de frutos, ya que están relacionados en la regulación de la floración (Berkovich et al., 2017; Demotes-Mainard et al., 2016).

\section{Concentración de pigmentos fotosintéticos y compues- tos fenólicos en hoja}

Los resultados obtenidos en la concentración de pigmentos fotosintéticos en hoja se muestran en la Tabla 3. De manera general se observó que las plantas que recibieron $0 \%$ de luz azul tuvieron mayor concentración de clorofila $a, b$ y total que el resto de tratamientos con luz LED, pero sin diferencias estadísticas en comparación al testigo y una disminución de concentración de carotenoides conforme la proporción de luz azul aumentó a partir de las plantas que recibieron $76 \%$ y $100 \%$ de luz azul en comparación con las plantas que solo recibieron luz roja. 
Tabla 3. Pigmentos fotosintéticos y compuestos fenólicos en hojas de chile habanero cultivado bajo condiciones controladas con diferentes proporciones de luz LED azul y roja.

Table 3. Photosynthetic pigments and phenolic compounds in habanero pepper leaves grown under controlled conditions with different blue and red LED light proportions.

\begin{tabular}{lcccccc}
\hline & \multicolumn{5}{c}{ Tratamientos $^{\mathrm{x}}$} \\
\cline { 2 - 7 } Variables & $\mathbf{F L}$ & $\mathbf{A 0}$ & $\mathbf{A 3 4 . 5}$ & $\mathbf{A 5 7 . 1}$ & $\mathbf{A 7 6 . 0}$ & $\mathbf{A 1 0 0}$ \\
\hline $\mathrm{Cla}$ & $0.90 \pm 0.08 \mathrm{ab}$ & $1.03 \pm 0.03 \mathrm{a}$ & $0.68 \pm 0.04 \mathrm{~b}$ & $0.74 \pm 0.06 \mathrm{ab}$ & $0.65 \pm 0.12 \mathrm{~b}$ & $0.65 \pm 0.06 \mathrm{~b}$ \\
$\mathrm{Clb}$ & $0.50 \pm 0.02 \mathrm{ab}$ & $0.58 \pm 0.02 \mathrm{a}$ & $0.40 \pm 0.02 \mathrm{bc}$ & $0.39 \pm 0.02 \mathrm{bc}$ & $0.35 \pm 0.05 \mathrm{c}$ & $0.33 \pm 0.03 \mathrm{c}$ \\
\hline Cltot & $1.40 \pm 0.10 \mathrm{ab}$ & $1.61 \pm 0.04 \mathrm{a}$ & $1.09 \pm 0.06 \mathrm{~b}$ & $1.14 \pm 0.08 \mathrm{~b}$ & $1.00 \pm 0.17 \mathrm{~b}$ & $0.98 \pm 0.08 \mathrm{~b}$ \\
$\mathrm{Cla} / \mathrm{b}$ & $1.81 \pm 0.09^{\mathrm{ns}}$ & $1.77 \pm 0.03^{\mathrm{ns}}$ & $1.68 \pm 0.02^{\mathrm{ns}}$ & $1.87 \pm 0.04^{\mathrm{ns}}$ & $1.81 \pm 0.08^{\mathrm{ns}}$ & $1.93 \pm 0.03^{\mathrm{ns}}$ \\
\hline Car & $4.20 \pm 0.40 \mathrm{ab}$ & $4.53 \pm 0.04 \mathrm{a}$ & $3.16 \pm 0.18 \mathrm{ab}$ & $3.43 \pm 0.22 \mathrm{ab}$ & $2.93 \pm 0.47 \mathrm{~b}$ & $2.93 \pm 0.24 \mathrm{~b}$ \\
CF & $29.14 \pm 1.97 \mathrm{~b}$ & $39.27 \pm 1.14 \mathrm{ab}$ & $29.86 \pm 3.58 \mathrm{~b}$ & $31.23 \pm 2.52 \mathrm{~b}$ & $40.93 \pm 3.04 \mathrm{ab}$ & $53.36 \pm 4.75 \mathrm{a}$ \\
\hline
\end{tabular}

${ }^{\times}$Medias con igual letra en las filas son estadísticamente iguales (Tukey, $\left.\mathrm{P} \leq 0.05\right)$. $\mathrm{ns}=$ No significativo; $\mathrm{FL}=$

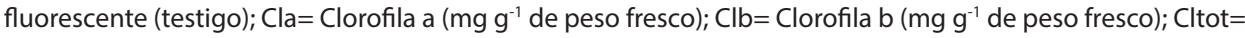
Clorofila total ( $\mathrm{mg} \mathrm{g}^{-1}$ de peso fresco); $\mathrm{Cla} / \mathrm{b}=\mathrm{Clorofila} \mathrm{a} / \mathrm{b} ; \mathrm{Car}=$ Carotenoides ( $\mathrm{mg} \mathrm{g}^{-1}$ de peso fresco); $\mathrm{CF}=$ Fenoles totales ( $\mathrm{mg}$ equivalentes de ácido gálico $100 \mathrm{~g}$ de peso fresco).

Liu et al. (2018) encontraron en plántulas de tomate un comportamiento similar de clorofila a, b y total, en plantas que recibieron $100 \%$ de luz azul, éstas obtuvieron la menor concentración en comparación con las que tuvieron 75 $\%, 60 \%, 50 \%$ y $25 \%$ de luz respecto a luz roja. De manera contraria, Wang et al. (2016) observaron en lechuga un comportamiento contrario, en donde plantas que recibieron solo luz roja monocromática obtuvieron la menor concentración de clorofila total, en comparación con las que recibieron 100 $\%, 50 \%, 20 \%, 11 \%$ de luz azul respecto a la luz roja. Agarwal et al. (2018) encontraron en espinaca un comportamiento similar a nuestros resultados respecto a la concentración de carotenoides.

El comportamiento de los pigmentos fotosintéticos en hoja, podrían explicarse en base a que la presencia de luz azul tiende a disminuir la clorofila total (Huché-Thélier et al., 2016), y además los fitocromos, asociados a la luz roja, regulan las etapas tempranas de biosíntesis de clorofila y carotenoides (Berkovich et al., 2017). Las plantas presentan un alto grado de plasticidad a las condiciones de luz que son expuestas, ya que al crecer bajo luz LED azul y roja, nunca recibieron longitudes de onda superiores a $680 \mathrm{~nm}$, con lo cual el fotosistema II $(680 \mathrm{~nm})$ estará, preferentemente excitado en relación al fotosistema I $(700 \mathrm{~nm})$ durante todo el ciclo de crecimiento, esto no sucede en la naturaleza, pero aun así las plantas crecen y posen mecanismos de aclimatación a largo plazo que aún no se han determinado (Pocock, 2015).

La concentración de compuestos fenólicos en hojas favoreció su acumulación en plantas que tuvieron un tratamiento monocromático azul en comparación con las que recibieron $34.5 \%$ y $57.1 \%$ de luz azul y con el testigo (Tabla 3). Jeon et al. (2017) encontraron en Oenanthe stolonifera un comportamiento similar en la concentración de compuestos fenólicos en donde utilizaron tratamientos de luz monocromática azul y roja, al igual que diferentes proporciones de éstos.

La luz azul tiende a incrementar la síntesis y acumulación de compuestos fenólicos (Berkovich et al., 2017), debido a que es un mecanismo de tolerancia al estrés foto-oxidativo y puede asumirse que está regulado por la producción de éste a través del fotorreceptor criptocromo, el cual está asociado con la foto activación a la luz azul (400 a $500 \mathrm{~nm}$ ) y UV-A (320 a 400 nm) (Huché-Thélier et al., 2016).

La presencia de luz azul y roja pueden ser responsables del aumento de la producción de metabolitos secundarios de la planta, debido a un aumento en la actividad de la enzima fenilalanina amonio liasa (PAL) la cual está involucrada en el primer paso de la vía de síntesis de fenilpropanoides (Hasan et al., 2017). La enzima PAL se asocia con la producción de compuestos fenólicos en plantas, la cual es mayormente estimulada bajo la combinación de luz azul y roja (Heo et al., 2012). Sin embargo, el comportamiento de los resultados puede variar debido a que no solo depende del tipo del espectro de luz que inciden en las plantas, si no, también depende de la especie, la variedad y etapa de desarrollo (Samuolienè et al., 2012).

\section{Concentración nutrimental en hoja}

En la Tabla 4, se muestran los resultados que se obtuvieron de nutrientes en hoja chile habanero. En los macronutrientes, los diferentes tratamientos de luz no ejercieron efecto en la concentración de P en hoja, sin embargo, el tratamiento de luz monocromática roja presentó la menor concentración. Entre tratamientos con luz LED no existió diferencias estadísticas en la concentración de K, sin embargo, la concentración de $\mathrm{K}$ en las plantas del testigo fue mayor en comparación con las plantas que recibieron $0 \%$ y $76 \%$ de luz azul. La concentración de Mg en hoja fue mayor en las plantas que recibieron $0 \%$ de luz roja en comparación con el resto de tratamientos LED y el testigo.

En los micronutrientes, la concentración de $\mathrm{Cu}$ en hojas de plantas del testigo y que recibieron $100 \%$ de azul, fue mayor que las que recibieron solo luz monocromática roja. La concentración de $\mathrm{Mn}$, fue mayor las hojas de las plantas que recibieron $0 \%$ de luz azul que las que recibieron $100 \%$ y 57.1 $\%$ de luz azul como además que las del testigo. 
Tabla 4. Concentración nutrimental en hojas de chile habanero cultivado bajo condiciones controladas con diferentes proporciones de luz LED azul y roja.

Table 4. Nutrient concentration in habanero pepper leaves grown under controlled conditions with different blue and red LED light ratios.

\begin{tabular}{lcccccc}
\hline \multicolumn{5}{c}{ Tratamientos $^{\mathbf{x}}$} \\
\cline { 2 - 7 } Ele & $\mathbf{F L}$ & $\mathbf{A 0}$ & $\mathbf{A 3 4 . 5}$ & $\mathbf{A 5 7 . 1}$ & $\mathbf{A 7 6 . 0}$ & $\mathbf{A 1 0 0}$ \\
\hline $\mathrm{N}$ & $4.69 \pm 0.07^{\text {ns }}$ & $4.08 \pm 0.10^{\text {ns }}$ & $4.81 \pm 0.42^{\text {ns }}$ & $4.83 \pm 0.25^{\text {ns }}$ & $5.30 \pm 0.30^{\text {ns }}$ & $4.33 \pm 0.59^{\text {ns }}$ \\
\hline $\mathrm{P}$ & $0.47 \pm 0.00 \mathrm{a}$ & $0.33 \pm 0.00 \mathrm{~b}$ & $0.52 \pm 0.03 \mathrm{a}$ & $0.49 \pm 0.03 \mathrm{a}$ & $0.45 \pm 0.00 \mathrm{a}$ & $0.53 \pm 0.02 \mathrm{a}$ \\
$\mathrm{K}$ & $5.20 \pm 0.06 \mathrm{a}$ & $3.62 \pm 0.10 \mathrm{~b}$ & $3.92 \pm 0.41 \mathrm{ab}$ & $4.14 \pm 0.42 \mathrm{ab}$ & $3.75 \pm 0.19 \mathrm{~b}$ & $4.00 \pm 0.28 \mathrm{ab}$ \\
$\mathrm{Ca}$ & $1.12 \pm 0.05^{\text {ns }}$ & $1.41 \pm 0.07^{\text {ns }}$ & $1.34 \pm 0.10^{\text {ns }}$ & $1.32 \pm 0.05^{\text {ns }}$ & $1.25 \pm 0.08^{\text {ns }}$ & $1.40 \pm 0.10^{\text {ns }}$ \\
\hline $\mathrm{Mg}$ & $0.74 \pm 0.01 \mathrm{~b}$ & $1.30 \pm 0.07 \mathrm{a}$ & $0.92 \pm 0.12 \mathrm{~b}$ & $0.68 \pm 0.02 \mathrm{~b}$ & $0.89 \pm 0.06 \mathrm{~b}$ & $0.68 \pm 0.03 \mathrm{~b}$ \\
$\mathrm{~B}$ & $49.56 \pm 0.62^{\text {ns }}$ & $52.37 \pm 1.95^{\text {ns }}$ & $51.47 \pm 0.96^{\text {ns }}$ & $46.47 \pm 1.46^{\text {ns }}$ & $50.54 \pm 3.72^{\text {ns }}$ & $59.40 \pm 5.54^{\text {ns }}$ \\
\hline $\mathrm{Fe}$ & $223.33 \pm 26.42^{\text {ns }}$ & $166.25 \pm 13.94^{\text {ns }}$ & $255.30 \pm 57.70^{\text {ns }}$ & $198.93 \pm 8.19^{\text {ns }}$ & $193.44 \pm 3.03^{\text {ns }}$ & $244.12 \pm 22.94^{\text {ns }}$ \\
\hline $\mathrm{Cu}$ & $11.23 \pm 0.62 \mathrm{a}$ & $5.24 \pm 0.18 \mathrm{c}$ & $9.85 \pm 0.93 \mathrm{ab}$ & $10.00 \pm 0.61 \mathrm{ab}$ & $7.91 \pm 0.37 \mathrm{bc}$ & $10.78 \pm 0.59 \mathrm{a}$ \\
\hline $\mathrm{Mn}$ & $96.850 \pm 14.41 \mathrm{bc}$ & $171.20 \pm 8.61 \mathrm{a}$ & $136.51 \pm 8.40 \mathrm{ab}$ & $89.02 \pm 7.00 \mathrm{bc}$ & $118.11 \pm 18.96 \mathrm{abc}$ & $77.98 \pm 1.04 \mathrm{c}$ \\
\hline $\mathrm{Zn}$ & $136.69 \pm 7.51^{\text {ns }}$ & $119.93 \pm 16.43^{\text {ns }}$ & $188.35 \pm 34.12^{\text {ns }}$ & $147.58 \pm 18.61^{\text {ns }}$ & $119.88 \pm 12.48^{\text {ns }}$ & $148.14 \pm 1.58^{\text {ns }}$ \\
\hline
\end{tabular}

${ }^{\times}$Medias con letras iguales en las filas son estadísticamente iguales (Tukey, $\left.\mathrm{P} \leq 0.05\right)$. Ele= Elementos; $\mathrm{ns}=$ No significativo; $\mathrm{FL}=$ fluorescente (testigo); $\mathrm{N}=$ Nitrógeno (\% de peso seco); $\mathrm{K}=$ Potasio (\% de peso seco); $\mathrm{P}=$ Fósforo (\% de peso seco); $\mathrm{Ca}=$ Calcio (\% de peso seco); $\mathrm{Mg}=$ Magnesio (\% de peso seco); $\mathrm{Fe}=$ Hierro $\left(\mathrm{mg} \mathrm{kg}^{-1}\right.$ de peso seco); $\mathrm{Mn}=$ Manganeso $\left(\mathrm{mg} \mathrm{kg}^{-1} \mathrm{de}\right.$ peso seco); $\mathrm{Zn}=\mathrm{Zinc}\left(\mathrm{mg} \mathrm{kg}^{-1}\right.$ de peso seco); $\mathrm{B}=$ Boro $\left(\mathrm{mg} \mathrm{kg}^{-1}\right.$ de peso seco); $\mathrm{Cu}=\mathrm{Cobre}\left(\mathrm{mg} \mathrm{kg}^{-1}\right.$ de peso seco).

Pennisi et al. (2019) encontraron en albahaca una tendencia de mayor acumulación de nutrientes $(\mathrm{N}, \mathrm{P}, \mathrm{K}, \mathrm{Ca}, \mathrm{Mg}$, $\mathrm{Fe}$ ) con una proporción de luz azul y roja A23 \%:R70 \%. De manera contraria, Verma et al. (2018) registraron en digitales que una proporción de luz azul y roja A80 \%:R20 \% obtuvo la máxima concentración de nutrientes $(\mathrm{P}, \mathrm{S}, \mathrm{Ca}, \mathrm{Mg}, \mathrm{Fe}, \mathrm{Mn}, \mathrm{Zn}$, $\mathrm{Zn}, \mathrm{B}, \mathrm{Cu}$ ).

La regulación de nutrientes en plantas está asociado a factores como la especie, luz, temperatura, $\mathrm{CO}_{2}$ ambiental, estrés hídrico, fitohormonas y la presencia de otros nutrientes, debido a un posible mecanismo de optimización de crecimiento al estar en diferentes condiciones de crecimiento (Sakuraba y Yanagisawa, 2017).

Sakuraba et al. (2018) mencionan que la luz roja promueve la absorción y acumulación de fósforo en Arabidopsis debido a que el fitocromo $B$ media el mecanismo regulatorio de absorción y uso de fósforo en la planta, a través de dos vías de señalización y regulación asociadas a la luz roja: víafitocromo B-HY5 y vía-fitocromo B-PIFs (PHYTOCHROME INTERACTING FACTOR family), los cuales en conjunto inducen la expresión del gen transportador de fósforo inorgánico ubicado en raíz PHT1;1 (INORGANIC PHOSPHATE TRANSPORTER) $y$ el gen PHL1 (HOMEODOMAIN-LIKE SUPERFAMILY PROTEIN) el cual induce también la expresión del gen PHT1;1.

La menor concentración de fósforo en hoja se registró en plantas que recibieron solo luz roja (Tabla 4), una posibilidad de esto pudo deberse a la alta demanda de nutrientes y fotosintatos a la cual la planta estaba sometida, debido a que tuvo una mayor cantidad de frutos respecto al resto de tratamientos.

García-Caparrós et al. (2019) mencionan que la concentración de potasio en plántulas de Arabidopsis se incrementó con luz azul debido a cambios en la actividad de transporte de la membrana en células guarda en estomas. Este comportamiento no se observó en ningún tratamiento con luz LED en plantas de habanero, sin embargo, se puede asumir que otras longitudes de onda pudieran estar involucradas en la absorción de potasio, dado que en las plantas que recibieron luz fluorescente, el cual tiene un espectro completo que abarca desde los 416 - $715 \mathrm{~nm}$, se obtuvo mayor concentración en comparación que las plantas que recibieron $0 \%$ y $76 \%$ de luz azul.

El mecanismo que modula el contenido de cobre en cloroplastos se asocia con el factor de transcripción HY5, el cual es activado por la señalización generada de diversos fotoreceptores que son activados por el tipo de luz asociada a éstos, posteriormente el factor de transcripción HY5 interactúa físicamente con el factor de transcripción SPL7 (SQUAMOSA PROMOTER-BINDING PROTEIN-LIKE 7), los cuales coactivan el gen MIR408 (microRNA408) que es requerido para regular la expresión de los genes PETE1 (PLASOCYANIN 1) y PETE2 (PLASTOCYANIN 2), encargados de la síntesis de la proteína plastocianina que contiene cobre en su estructura y se ubica en cloroplastos (Gangappa y Botto, 2016; Sakuraba y Yanagisawa, 2017). Por alusión a lo anterior, en el tratamiento monocromático azul se promovió la síntesis de plastocianina asociada a cobre en plantas de habanero en comparación con las plantas que recibieron luz roja monocromática.

Miao et al. (2019) mencionan que la luz roja promueve la acumulación de manganeso, lo cual coincidió con lo obtenido en planta de habanero en el tratamiento monocromático rojo en comparación con los resultados del tratamiento monocromático azul.

La concentración de nutrientes en tejidos de plantas puede ser modificada por factores específicos como la transpiración, apertura estomática, tasa de crecimiento y acumu- 
lación de biomasa en diferentes etapas de desarrollo (Pennisi et al., 2019). Se ha demostrado que la luz azul está involucrada en el proceso de acumulación de nutrientes en planta a través de mecanismos que incrementan la activación de las bombas de protones y la actividad de canales iónicos en membranas celulares, con lo cual se incrementa la actividad de transporte de iones en las membranas celulares en los tejidos de plantas (Gerovac et al., 2016). La luz azul tiene además la capacidad de regular la apertura estomática, lo cual, junto con la capacidad que tiene de incrementar el transporte de iones a través de las membranas celulares, puede afectar directamente la absorción de nutrientes mediante un aumento en la transpiración de la planta (Craver et al., 2018). Existe poca información sobre como ciertas longitudes de onda pueden afectar la dinámica de absorción de nutrientes en plantas, por lo cual se requiere mayor investigación para descifrar estos mecanismos (Amoozgar et al., 2016).

\section{Variables anatómicas y viabilidad de polen}

Los cortes anatómicos mostraron la existencia de óvulos bien desarrollados dentro de los ovarios, en base a la presencia de óvulos dentro del ovario, pared de ovario, núcleo del ovulo y tegumentos en todos los tratamientos evaluados. Por otra parte, hubo la presencia de polen maduro viable en anteras, en base a la existencia de una tinción completa del citoplasma del polen y de color rojo intenso en casi todos los granos de polen que se observaron en los cortes anatómicos a lo largo de todos los tratamientos evaluados (Figura 1). Estos resultados coinciden con la descripción descrita por Pérez-Pastrana et al. (2018) en cortes anatómicos de flores en antesis para la misma variedad de chile habanero.

El mayor porcentaje de germinación de granos de polen se presentó en las plantas del testigo, sin embargo, no existió diferencias entre los diferentes tratamientos con luz LED (Tabla 5). Dhawan y Malik (1981) estudiaron la germinación de polen in vitro en Pinus roxburghii con diferentes tipos de luz y encontraron el menor porcentaje con luz fluorescente $(40 \%)<$ luz rojo + rojo lejano $(40 \%)<$ luz azul $(60 \%)<$ oscuridad (60 \%) <luz roja (95\%) y luz roja con el máximo de germinación. Hoyo et al. (2014) investigaron la germinación de polen in vitro en Cyrtanthus mackenii con cinco picos de longitud de onda, $405 \mathrm{~nm}$ (violeta), $465 \mathrm{~nm}$ (azul), $630 \mathrm{~nm}$ (rojo naranja), $660 \mathrm{~nm}$ (rojo), $735 \mathrm{~nm}$ (rojo lejano) y oscuridad, concluyendo que la luz no inhibió o promovió la germinación de polen ni tampoco las longitudes de onda afectaron la tasa de germinación.
Lo anterior sugiere que el efecto del tipo de luz sobre la germinación de polen depende de la especie; además, en chile habanero, los resultados en los tratamientos LED no presentaron diferencias estadísticas entre éstos. Por otra parte, la luz fluorescente al ser una fuente de luz de espectro completo $(416-715 \mathrm{~nm})$, promovió el porcentaje de germinación, probablemente a que posee una mayor diversidad de longitudes de onda en comparación con el resto de los tratamientos, e incluso a pesar de que la intensidad de la luz fluorescente fue la mitad en relación a estos mismos tratamientos de luz LED.

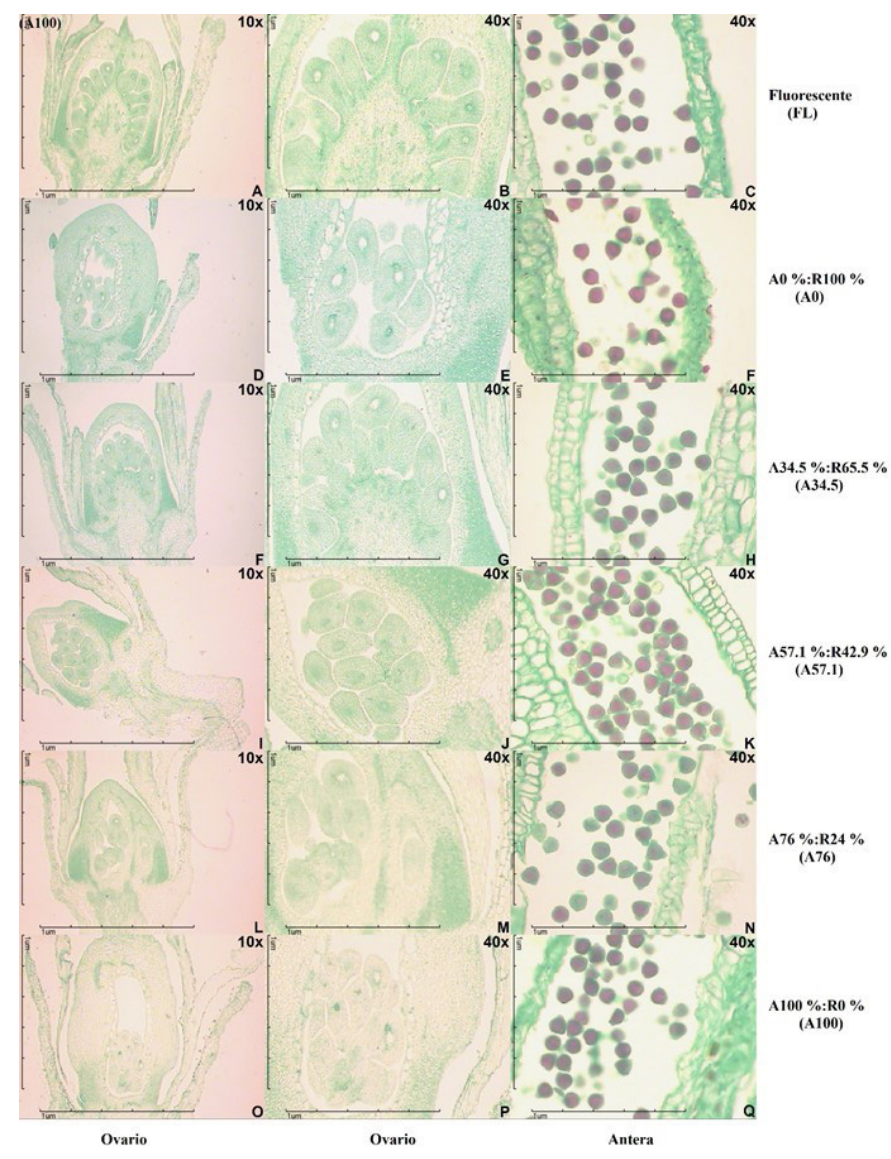

Figura 1. Cortes anatómicos longitudinales de ovario y polen de anteras de flores de chile habanero cultivado bajo condiciones controladas con diferentes proporciones de luz LED azul y roja.

Figure 1. Anatomical longitudinal sections of ovary and flower anther pollen of habanero pepper grown under controlled conditions with different blue and red LED light ratios.

Tabla 5. Germinación de polen y grosor de hoja en chile habanero cultivado bajo condiciones controladas con diferentes proporciones de luz LED azul y roja.

Table 5. Pollen germination and leaf thickness on habanero pepper grown under controlled conditions with different blue and red LED light ratios.

\begin{tabular}{lcccccc}
\hline & \multicolumn{5}{c}{ Tratamientos $^{\mathrm{x}}$} \\
\cline { 2 - 7 } Variables & FL & A0 & A34.5 & A57.1 & A76.0 & A100 \\
GP & $44.83 \pm 5.65 \mathrm{a}$ & $17.95 \pm 4.11 \mathrm{~b}$ & $4.30 \pm 1.76 \mathrm{~b}$ & $0.28 \pm 0.29 \mathrm{~b}$ & $12.17 \pm 6.57 \mathrm{~b}$ & -- \\
GH & $115.21 \pm 5.1 \mathrm{a}$ & $74.17 \pm 3.2 \mathrm{~d}$ & $93.89 \pm 3.66 \mathrm{bc}$ & $76.67 \pm 2.43 \mathrm{~cd}$ & $94.72 \pm 2.90 \mathrm{~b}$ & $115.00 \pm 6.5 \mathrm{a}$ \\
\hline
\end{tabular}

'Medias con letras iguales en las filas son estadísticamente iguales (Tukey, $\mathrm{P} \leq 0.05)$. $\mathrm{ns}=$ No significativo; $\mathrm{FL}=$ fluorescente (testigo); Var=Variables; $\mathrm{GP}=$ Germinación de polen (\%); $\mathrm{GH}=$ Grosor de hoja $(\mu \mathrm{m})$. 
El grosor de hoja fue mayor tanto en las plantas del testigo como las que recibieron $100 \%$ de luz azul en comparación con las que recibieron luz monocromática roja y las que tuvieron $76 \%$ de luz azul (Tabla 5). Wollanger y Runkle (2015) encontraron un comportamiento similar en salvia, ya que el grosor de hoja fue mayor en un tratamiento monocromático azul respecto a uno de rojo.

En chile habanero, el diferente grosor de hoja entre tratamientos, pudo deberse a que la luz azul tiende a incrementar el grosor de hoja respecto a la luz roja (Huché-Thélier et al., 2016). Evert y Eichhorn (2012) mencionan que, a mayor intensidad de luz, las hojas tienden a tener un mayor grosor $y$ viceversa; sin embargo, en el testigo a pesar de tener la mitad de intensidad luminosa respecto a los tratamientos LED tuvo el mayor grosor de hoja junto con las plantas que recibieron luz azul monocromática.

\section{CONCLUSIONES}

Los tratamientos evaluados no indujeron un efecto en las variables de crecimiento. La presencia de luz LED roja indujo el amarre y desarrollo de los frutos, además, favoreció la frecuencia estomática.

La luz roja monocromática favoreció la acumulación de pigmentos fotosintéticos, mientras que la luz azul monocromática indujo mayor concentración de compuestos fenólicos en hoja en la mayoría de los tratamientos evaluados.

La luz roja monocromática indujo menor concentración $\mathrm{P}$, y favoreció la concentración de Mg. La luz LED no generó efecto en la concentración de K, sin embargo, el testigo indujo mayor concentración en comparación con algunos tratamientos. La luz monocromática roja indujo menor concentración de $\mathrm{Cu}$ en hoja e indujo en Mn, mayor concentración en comparación con otros tratamientos evaluados.

El testigo incrementó la germinación de los granos de polen y el grosor de hoja, igual que la luz azul en esta última variable. La anatomía de los óvulos en ovarios y el polen en anteras no presentaron ningún efecto de los tratamientos.

\section{REFERENCIAS}

Amoozgar, A., Mohammadi, A. y Sabzalian, M.R. 2016. Impact of light-emitting diode irradiation on the photosynthesis, phytochemical composition and mineral element content of lettuce cv. Grizzly. Photosynthetica. 55: 85-95. https://doi. org/ 10.1007/s11099-016-0216-8

Alcántar, G. y Sandoval, M. 1999. Manual de análisis químico de tejido vegetal. Publicación especial 10. Sociedad Mexicana de la Ciencia del Suelo, A. C. Estado de México, México.

Agarwal, A., Gupta, S.D., Barman, M. y Mitra, A. 2018. Photosynthetic apparatus plays a central role in photosensitive physiological acclimations affecting spinach (Spinacia oleracea L.) growth in response to blue and red photon flux ratios. Environmental and Experimental Botany. 156: 170-182. https://doi.org/10.1016/j. envexpbot.2018.09.009

AOAC. 1980. Official methods of analysis. 12th ed. Association of Official Analytical Chemistry. Washington, D.C.

Berkovich, Y.A., Konovalova, I.O., Smolyanina, S.O., Erokhin, A.N., Avercheva, O.V., Bassarskaya, E.M., Kochetova, G.V.,
Zhigalova, T.V., Yakovleva, O.S. y Tarakanov, I.G. 2017. LED crop illumination inside space greenhouses. REACH - Reviews in Human Space Exploration. 6: 11-24. http:// dx.doi.org/10.1016/j.reach.2017.06.001

Blankenship, R.E.2014. Molecularmechanisms of photosynthesis. 2nd ed. Wiley Blackwell. New Delhi, India.

Bojórquez-Quintal, E., Velarde-Buendía, A., Ku-González, A., Carillo-Pech, M., Ortega-Camacho, D., Echevarría-Machado, I., Pottosin I. y Martínez-Estévez, M. 2014. Mechanisms of salt tolerance in habanero pepper plants (Capsicum chinense Jacq.): proline accumulation, ions dynamics and sodium root-shoot partition and compartmentation. Frontiers in Plant Science. 5: 605. http://dx.doi.org/10.3389/ fpls.2014.00605

CICY. 2016. Ficha informativa unidad productora de semillas de chile habanero. Centro de Investigación Científica de Yucatán. Abril (2016): 1-15. [Consultado 10 enero 2020]. Disponible en: https://www.cicy.mx/Documentos/CICY/ quienes-somos/2016/Ficha-UPS-Habanero.pdf

Craver, J.K., Boldt, J.K. y Lopez, R.G. 2018. Radiation intensity and quality from sole-source light-emitting diodes affect seedling quality and subsequent flowering of long-day bedding plant species. HortScience. 53(10): 1407-1415. https://dx.doi.org/ 10.21273/HORTSCI13228-18

Dhawan, A.K. y Malik, C.P. 1981. Effect of growth regulators and light on pollen germination and pollen tube growth in Pinus roxburghii Sarg. Annals of Botany. 47(2): 239-248.

Demotes-Mainard, S., Pérona, T., Corotb, A., Bertheloota, J., Gourrierecb, J.L., Pelleschi-Travierb, S., Crespel, L., Morel, P., Huché-Thélier, L., Boumaza, R., Vian, A., Guérin, V., Leduc, N. y Sakr, S. 2016. Plant responses to red and far-red lights, applications in horticulture. Environmental and Experimental Botany. 121: 4-21. http://dx.doi.org/10.1016/j. envexpbot.2015.05.010

Evert, R.F. y Eichhorn, S.E. 2012. Raven biology of plants. 8 ed. MacMillan, USA.

Gangadhar, B.H., Mishra, R.K., Pandian, G. y Park, S.W. 2012. Comparative study of color, pungency, and biochemical composition in chili pepper (Capsicum annuum) under different light-emitting diode treatments. Hortscience. 47(12): 1729-1735. https://doi.org/10.21273/ HORTSCI.47.12.1729

Gangappa, S.N. y Botto, J.F. 2016. The multifaceted roles of hy5 in plant growth and development. Molecular Plant. 9: 1353-1365. https://dx.doi.org/10.1016/j.molp.2016.07.002

García-Caparrós, P., Almansa, E.M., Chica, R.M. y Lao, M.T. 2019. Effects of artificial light treatments on growth, mineral composition, physiology, and pigment concentration in Dieffenbachia maculate "Compacta" plants. Sustainability. 11: 2867. https://dx.doi.org/10.3390/su11102867

Gerovac, J.R., Craver, J.K., Boldt, J.K. y Lopez, R.G. 2016. Light intensity and quality from sole-source light-emitting diodes impact growth, morphology, and nutrient content of Brassica microgreens. HortScience. 51(5): 497-503. https:// doi.org/10.21273/HORTSCI.51.5.497

Hasan, M., Bashir, T., Ghosh, R., Lee, S. K., y Bae, H. 2017. An overview of LEDs' effects on the production of bioactive compounds and crop quality. Molecules. 22(9): 1420. https:// dx.doi.org/10.3390/molecules22091420

Heo, J. W., Kang, D. H., Bang, H. S., Hong, S. G., Chun, C. H., y Kang, K. K. 2012. Early growth, pigmentation, protein content, and phenylalanine ammonia-lyase activity of red curled lettuces grown under different lighting conditions. Korean Journal 
of Horticultural Science and Technology. 30(1): 6-12. https:// dx.doi.org/10.7235/hort.2012.11118

Hoagland, D.R. y Arnon, D.I. 1950. The water-culture method for growing plants without soil. Circular 347. California Agricultural Experiment Station. California, USA.

Hogewoning, S.W., Trouwborst, G., Maljaars, H., Poorter, H., van leperen, W.y Harbinson, J. 2010. Blue light dose-responses of leaf photosynthesis, morphology, and chemical composition of Cucumis sativus grown under different combinations of red and blue light. Journal of Experimental Botany. 61(11): 3107-3117. http://dx.doi.org/10.1093/jxb/erq132

Hoyo, Y., Fujiwara, K. y Hoshino, Y. 2014. Effects of different wavelengths of LED light on pollen germination and direction of pollen tube elongation in Cyrtanthus mackenii. Advances in Horticultural Science. 28(4): 190-194.

Huché-Thélier, L., Crespel, L., Gourrierec, J.L., Morel, P., Sakr, S. y Leduc, N. 2016. Light signaling and plant responses to blue and UV radiations-Perspectives for applications in horticulture. Environmental and Experimental Botany. 121: 22-38. https://dx.doi.org/10.1016/j.envexpbot.2015.06.009

Jeon, Y., Son, K., Kim, S. y Oh, M. 2017. Growth and bioactive compounds as affected by irradiation with various spectrum of light-emitting diode lights in dropwort. Horticulture. Environment, and Biotechnology. 58: 467-478. https://doi. org/10.1007/s13580-017-0354-3

Katagiri, F., Canelon-Suarez, D., Griffin, K., Petersen, J., Meyer, R.K. y Siegle, M. 2015. Design and construction of an inexpensive homemade plant growth chamber. PLoS ONE. 10(5): e0126826. https://doi.org/10.1371/journal.pone.0126826

Kozai, T. y Niu, G. 2016. Role of the plant factory with artificial lighting (PFAL) in urban areas. En: Plant factory: An indoor vertical farming system for efficient quality food production. T. Kozai, G. Niu y M. Takagaki (ed.), pp 115-128. Academic Press, USA. https://dx.doi.org/10.1016/B978-0-12-8017753.00002-0

Kubota, C. 2016. Growth, development, transpiration and translocation as affected by abiotic environmental factors. En: Plant factory: An indoor vertical farming system for efficient quality food production. T. Kozai, G. Niu y M. Takagaki (ed.), pp 151-164. Academic Press, USA. http:// dx.doi.org/10.1016/B978-0-12-801775-3.00010-X

Lim, T. K. 2013. Capsicum chinense. En: Edible medicinal and non-medicinal plants: Volume 6, Fruits. T. K. Lim (ed.), pp 205-212. Springer Science+Business Media, Dordrecht. https://doi.org/10.1007/978-94-007-5628-1_30

Liu, X. Y., Jiao, X. L., Chang, T. T., Guo, S. R. y Xu, Z. G. 2018. Photosynthesis and leaf development of cherry tomato seedlings under different LED-based blue and red photon flux ratios. Photosynthetica. 56: 1212-1217. https://dx.doi. org/10.1007/s11099-018-0814-8

Miao, Y., Chen, Q., Qu, M., Gao, L. y Hou, L. 2019. Blue light alleviates 'red light syndrome' by regulating chloroplast ultrastructure, photosynthetic traits and nutrient accumulation in cucumber. Scientia Horticulturae. 257: 108680. https://doi.org/10.1016/j.scienta.2019.108680

Mercado, J.A., Fernández-Muñoz, R.F. y Quesada, M.A. 1994. In vitro germination of pepper pollen in liquid medium. Scientia Horticulturae. 57: 273-281. https://doi.org/10.1016/03044238(94)90110-4

Naznin, M.T., Lefsrud, M., Gravel, V. y Azad, M.O.K. 2019. Blue light added with red LEDs enhance growth characteristics, pigments content, and antioxidant capacity in lettuce, spinach, kale, basil, and sweet pepper in a controlled environment. Plants. 8(4): 93. https://doi.org/10.3390/ plants 8040093

Pennisi, G., Blasioli, S., Cellini, A., Maia, L., Crepaldi, A., Braschi, I., Spinelli, F., Nicola, S., Fernandez, J.A., Stanghellini, C., Marcelis, L.F.M., Orsini, F. y Gianquinto, G. 2019. Unraveling the role of red:blue LED lights on resource use efficiency and nutritional properties of indoor grown sweet basil. Frontiers in Plant Science. 10: 305. https://doi.org/10.3389/ fpls.2019.00305

Pérez-Pastrana, J., Islas-Flores, I., Bárány, I., Álvarez-López, D., Canto-Flick, A., Canto-Canché, B., Peña-Yama, L., MuñozRamírez, L., Avilés-Viñas, S., Testillano, P.S. y Santana-Buzzy, N. 2018. Development of the ovule and seed of Habanero chili pepper (Capsicum chinense Jacq.): Anatomical characterization and immunocytochemical patterns of pectin methyl-esterification. Journal of Plant Physiology. 230: 1-12. https://doi.org/10.1016/j.jplph.2018.08.005

Pocock, T. 2015. Light-emitting diodes and the modulation of specialty crops: light sensing and signaling networks in plants. HortScience. 50(9): 1281-1284. https://dx.doi. org/10.21273/HORTSCI.50.9.1281

Ruiz-Lau, N., Medina, F. y Martínez, M. 2011, El chile habanero: su origen y usos. Ciencia. Julio-Septiembre: 70-77.

Sakuraba, Y., Kanno, S., Mabuchi, A., Monda, K., Iba, K. y Yanagisawa, S. 2018. A phytochrome-B-mediated regulatory mechanism of phosphorus acquisition. Nature plants. 4: 1089-1101. https://dx.doi.org/10.1038/s41477-018-0294-7

Sakuraba, Y., y Yanagisawa, S. 2017. Light signalling-induced regulation of nutrient acquisition and utilisation in plants. Seminars in Cell \& Developmental Biology. 83: 123-132. https://doi.org/10.1016/j.semcdb.2017.12.014

Samuolienè, G., Sirtautas, R., Brazaitytè, A. y Duchovskis, P. 2012. LED lighting and seasonality effects antioxidant properties of baby leaf lettuce. Food Chemistry. 134(3): 1494-1499. http://dx.doi.org/10.1016/j.foodchem.2012.03.061

SAS Institute (2002) SAS System for Windows Computer Program. Software Version 9.0. Cary, North Carolina, USA.

Singleton, V.L. y Rossi, J.A. 1965. Colorimetry of total phenolics with phosphomolybdic-phosphotungstic acid reagents. American Society for Enology and Viticulture. 16: 144-158.

Trujillo, J.J.G. 2018. Caracterización de los recursos genéticos de Capsicum chinense Jacq. en la peninsula de Yucatán. En: Mejoramiento genético del chile habanero de la Península de Yucatán. T.A. González (ed.), pp 37-43. Centro de Investigación Científica de Yucatán. Mérida, México.

Verma, S.K., Gantait, S., Jeong, B.R. y Hwang, S.J. 2018. Enhanced growth and cardenolides production in Digitalis purpurea under the influence of different LED exposures in the plant factory. Nature Scientific Reports. 8: 18009. https://doi. org/10.1038/s41598-018-36113-9

Wang, J., Lu, W., Tong, Y. y Yang, Q. 2016. Leaf morphology, photosynthetic performance, chlorophyll fluorescence, stomatal development of lettuce (Lactuca sativa L.) exposed to different ratios of red light to blue light. Frontiers in Plant Science. 7: 250. https://doi.org/10.3389/fpls.2016.00250

Wollanger, H.M. y Runkle, E.S. 2015. Growth and acclimation of impatiens, salvia, petunia, and tomato seedlings to blue and red light. HortScience. 50(4): 522-529. https://doi. org/10.21273/HORTSCI.50.4.522

Yamori, W. 2016. Photosynthesis and respiration. En: Plant factory: An indoor vertical farming system for efficient quality food production. T. Kozai, G. Niu y M. Takagaki (ed.), pp 141-149. Academic Press, USA. https://dx.doi. org/10.1016/B978-0-12-801775-3.0009-3(LAI) 